\title{
Participación popular en la producción del hábitat. El caso del Área Metropolitana de Tucumán-Argentina ${ }^{1}$
}

\author{
Paula Boldrini² y Claudia Gómez López ${ }^{3}$
}

\begin{abstract}
RESUMEN
Los sectores populares urbanos asumen el proceso de satisfacción de la necesidad de hábitat mediante diferentes estrategias que incluyen la insurrección colectiva. Las condiciones de participación en los procesos de producción del hábitat pueden conocerse mediante la elaboración de estudios y análisis a distintas escalas. En la dinámica sociohistórica del aglomerado tucumano estos procesos pueden estudiarse en la expansión urbana ocurrida entre los años 1976 y 2008, analizada en este caso mediante un sistema de información geográfico. La estructura residencial urbana es la dimensión que permite comprender a escala metropolitana las condiciones a partir de las cuales se produce la participación social en la producción del hábitat popular. Los resultados de la investigación revelan como el proceso de toma de tierra es el mecanismo de participación capaz de liberar la acumulación de una necesidad de hábitat irresuelta en un contexto urbano excluyente.
\end{abstract}

Palabras clave: Participación, sectores populares, hábitat.

\begin{abstract}
The inhabitants of the urban slums in Tucuman struggle in pursuing good habitat conditions through various strategies including their collective insurrection. The conditions of participation in the described processes can be explored through research and analysis strategies at different scales. Regarding the socio-historical dynamics of the urban agglomerate in Tucuman, these processes can be studied by taking into account the urban expansion that occurred between 1976 and 2008 through the use of geographic information systems. The urban residential structure is the model for understanding metropolitan patterns of social participation in the process of production of popular habitat. This research reveals how appropriation of urban land is the mechanism for citizen participation in the context of urban exclusion.
\end{abstract}

Key words: Participation, popular sectors, habitat.

\footnotetext{
Artículo recibido el 27 de agosto de 2012, aceptado el 18 de junio de 2013 y corregido el 30 de julio de 2013.
}

\footnotetext{
2 Instituto de Desarrollo Urbano, Universidad Nacional de Tucumán (Argentina).

E-mail: paula_boldrini@hotmail.com

3 Facultad de Arquitectura y Urbanismo, Universidad Nacional de Tucumán (Argentina).

E-mail: claugolo@gmail.com
} 
Los procesos de resolución de necesidades sociales como la adquisición de la vivienda propia y la conformación del hábitat se desarrollaron mediante diferentes mecanismos de participación a lo largo de la historia, de las geografías y de las culturas. Se inician y desarrollan mediante la tarea particular de individuos, familias e incluso comunidades cuando se comprometen esfuerzos colectivos. A este histórico flujo de energías destinadas a la producción del hábitat, se contrapone la dificultad de acceso a los recursos tangibles necesarios como tierra y materiales, e intangibles como el acompañamiento técnico al proceso que permite potenciar esfuerzos. Estas carencias se han profundizado con el devenir histórico de la humanidad.

La disparidad entre la necesidad de hábitat, recursos y soluciones, promueve una situación de emergencia sociohabitacional que se remonta a la crisis de masificación de las ciudades derivada de la revolución industrial. Llega retardada y profundizada a América Latina, requiriendo -al igual que en el resto del mundo- la intervención del Estado para paliar la crisis habitacional $\left.\right|^{4}$. Se produce una apertura en el flujo de políticas públicas destinadas a la resolución del hábitat mediante producción de vivienda, infraestructura y equipamiento, en paralelo a esfuerzos autogestionados, conformando entre ambos la realidad urbana latinoamericana ${ }^{5}$.

Tanto la producción urbana del Estado como la autogestión suponen diferentes formas de participación social para la producción del hábitat.

\footnotetext{
4 En América Latina, como consecuencia de la industrialización por sustitución de importaciones, el problema habitacional emerge durante el $2^{\circ}$ tercio del s. XX. En Argentina, la primera crisis se plantea en Buenos Aires con la llegada de los inmigrantes y el surgimiento de barrios populares.

5 A estos flujos podemos agregar el sector privado. Este sector representa un grupo independiente -aunque en estrecha asociación con el Estado- que promueve acciones estructurantes de la conformación urbana de las ciudades actuales. Sin embargo, en el presente trabajo se opta por hacer foco sobre las dinámicas populares, por lo que se profundiza en aquellos aspectos vinculados al flujo de esfuerzos autogestionados y políticas públicas.
}

"En especial, los gobiernos locales latinoamericanos ensayaron diversas fórmulas de decisión de lo público o implementaron formas de gestión de programas y proyectos con una intencionalidad participativa, aunque con distinta suerte. Esto hace que la escala de la ciudad y los procesos participativos aparezcan muy asociados" (Quintar et al., 2010).

La intervención del Estado en la consolidación del hábitat popular es uno de los modos más característicos de producción de ciudad. Según Fernández Wagner (2006), es un proceso de gran magnitud en el que la toma de decisiones se encuentra centralizada en el Poder Ejecutivo, lo que le impide funcionar de manera local y efectiva bajo la ley, adoptando mecanismos donde la discrecionalidad es estructural, y la práctica está subordinada a la construcción del poder político en el territorio.

Tal como plantea López (2002), se trata de un proceso histórico de producción y reproducción del territorio en el que las mejoras son el fruto del juego dialéctico entre clases sociales. El Estado promueve y ejecuta políticas habitacionales para disminuir el conflicto social, y los sectores privados ejercen un rol protagónico en la producción del hábitat que se convierte en un proceso más de acumulación.

Clichevsky (1990) plantea que la insuficiencia e ineficacia estatal para dar respuesta a la masiva demanda de hábitat, contribuyó en Argentina y otros países latinoamericanos a que se active una nueva modalidad de construcción de hábitat autogestionado, correspondiente al modo de producción comunitario -más o menos organizado- a través de la ocupación directa de tierras por parte de los sectores populares. Estos mecanismos implican tanto la modalidad de invasión o tomas de tierras, como el resurgimiento del submercado ilegal de tierras.

Las tomas de tierras urbanas constituyen un fenómeno cosmopolita en América Latina surgido entre las décadas de 1960 y 1980, junto al explosivo crecimiento poblacional que desborda las ciudades existentes. La ocupación informal de tierras se produce debido a la imposibilidad de los sectores populares 
de acceder al suelo urbano a través del mercado formal. Superada la fase inicial de toma de tierra urbana o anexa a la ciudad, se inicia la de consolidación del barrio que representa costes aun superiores, y la tensión permanente entre los ocupantes y el aparato represivo del Estado (Davis, 2007).

La proliferación de programas de regularización y mejoramiento de villas evidencia la tendencia estatal a la consolidación de los procesos de ocupación y toma de tierras, como actitud conciliatoria frente a la incapacidad de implementar políticas preventivas respecto a los conflictos sociales emergentes $^{6}$. Sin embargo, estas disposiciones coexisten con la preservación del bien/suelo privado cuyo derecho garantiza, lo cual supone la proliferación de conflictos con los sectores populares y la opinión pública en general, en tanto las tomas de tierra se producen en acontecimientos de significativa violencia social.

La comunidad asume el proceso de satisfacción de la necesidad de hábitat mediante una insurrección colectiva, con diferentes condiciones de organización de acuerdo al caso. Las condiciones de participación popular en la producción del hábitat pueden conocerse mediante la elaboración de estudios y análisis a distintas escalas. En cada caso intervendrán diferentes variables a tener en cuenta para caracterizar de qué forma interviene o no la sociedad -y particularmente los sectores populares- en la configuración del hábitat. La escala barrial puede abordarse a partir del estudio de las relaciones internas que se construyen en un proceso colectivo (Boldrini, 2011), sin embargo, la escala urbana, que comprende al conjunto de barrios e incluso de ciudades miembros de un aglomerado, requiere de otro tipo de análisis. Precisa la identificación de condiciones urbanas, a través de las cuales inferir la manera en la

6 Un claro ejemplo es la ley provincial Corbalán que declaraba sujeto a expropiación y regularización los terrenos privados y provinciales donde existiera un asentamiento irregular hasta 1983. Con la aprobación de la Ley se buscaba sanear el problema habitacional, sin embargo, se vio desbordada por la demanda y el problema que, lejos de desaparecer fue cada vez más acuciante. que los diferentes grupos sociales intervienen en el proceso de conformación urbana.

Sin limitar el debate de la participación al campo exclusivo del análisis netamente urbano, este trabajo plantea mostrar el contexto en el que se desarrolla la participación social en los procesos de producción de hábitat local. La ciudad o el conjunto de ciudades son entendidas como sostén de estos procesos, escenarios de implosión de estas necesidades sin resolución en el funcionamiento de la dinámica urbana.

El estudio de la estructura residencial urbana es el abordaje elegido para cumplir con el objetivo propuesto: caracterizar la participación social en la producción del hábitat popular del Área Metropolitana de Tucumán (en adelante, AMET). De esta forma se abordan las conexiones entre las estrategias participativas y la configuración de la ciudad, en un contexto en el que si bien se promueven las prácticas participativas, aún no se desarroIlan estrategias apropiadas para contrarrestar la inercia tradicional de producción del hábitat, en la que los sectores populares carecen de poder concreto en la elección de su localización residencial (Boldrini, 2012).

El análisis residencial diacrónico de la configuración del Área Metropolitana de Tucumán sirve para comprender las condiciones de participación de los sectores populares en el proceso de conformación urbana. Se trata de develar las lógicas participativas mediante las cuales se configuran los asentamientos informales en la ciudad, teniendo en cuenta su relación con otros sectores sociales con los que, en conjunto, se configura la dinámica sociohistórica del aglomerado tucumano. Se considera la expansión urbana entre los años 1976 y 2008.

La premisa de este artículo radica en que no existe la participación plena en el proceso de conformación urbana en tanto los habitantes pertenecientes a sectores populares deben ocupar espacios vulnerables que -de haber tenido la posibilidad- no hubieran elegido para vivir. Esta es una de las causas que suscitan tomas de tierra que irrumpen como respuesta a la falta de contención urbana y como mecanismo de pulsión hacia la búsque- 
da de participación para la satisfacción de la necesidad de hábitat.

Entendemos esta "posibilidad" como las condiciones concretas de acceso al suelo urbano, que superan la letra escrita de los derechos constitucionales ${ }^{7}$, para constituirse como capacidad o libertad plena de acceso concreto a tierras saneadas, urbanizadas o aptas para ser urbanizadas, considerando, incluso, a las tierras de elevado valor ambiental. Sin embargo, la protesta representa un aspecto emergente que da cuenta de las intensas pujas que se producen para acceder al suelo $y$, en cierta forma, ser parte en la definición de la configuración de la ciudad en lugar de ocupar los terrenos de descarte inmobiliario (Boldrini, 2012).

\section{La participación popular en la producción de la ciudad}

La participación es un concepto clave para la producción del hábitat, amplia e internacionalmente difundido en la actualidad. Hoy en día resulta imposible desvincular la participación social de cualquier tipo de política pública estatal, y por lo tanto de su reconocimiento como instrumento requerido para todo proceso social que pretenda identificarse como democrático (Borja, 1998).

Por su parte, Yory (2003) define la participación como la interacción entre grupos o personas en procesos de construcción de bienes colectivos. La ciudad puede entenderse como un bien colectivo conformado a partir de las acciones de los sujetos actuantes desde los diferentes grupos sociales.

"Sin lugar a dudas, el tema de la planificación participativa es uno de los más importantes en la vía de promover el fortalecimiento en las condiciones de gobernabilidad de los territorios y, desde aquí, en la promoción de pactos y compromi-

\footnotetext{
7 Por ejemplo "Las leyes que regulan los loteos urbanos dejan en manos de los particulares la fijación de precios sin que se prevean mecanismos de regulación estatal que puedan compensar los desvíos del mercado e incluir a la demanda con menor capacidad de pago" (Di Lullo, Giobellina, 1996:87).
}

sos multiactorales abocados a la construcción de un espíritu de convivencia que permita hacer de las ciudades un acontecimiento colectivo donde las diferencias no solo tengan lugar, sino que, de hecho, cuenten con espacio para el concierto" (Yory, 2003: 756).

Sin embargo, la realidad expresa condiciones que hacen de la planificación participativa una meta aún muy lejana. Las ciudades latinoamericanas presentan condiciones muy similares al reproducir ciertos aspectos claves como la fragmentación socioespacial. La fragmentación conlleva desigualdad (Sabatini, 2003), y se sustenta en sistemas políticos, sociales y económicos-productivos consecuentes con un modelo espacial desequilibrado (Yory, 2003). Prévôt Schapira (2001) describe a este nuevo modelo de ciudad fragmentada, como aquella en la que prevalece la pérdida de continuidad espacial dada por la existencia de fragmentos o partes aisladas de un todo, que tiene como situaciones extremas a la riqueza y la pobreza urbana.

Las urbanizaciones cerradas y las urbanizaciones no consolidadas representan, en la ciudad latinoamericana, las antípodas del hábitat neoliberal. El Estado no logra con su intervención mediar o equilibrar el modelo focalizando su actuación en aquellas franjas sociales que pueden satisfacer los requerimientos de "formalidad" que impone el sistema.

Los polos son la expresión de una sociedad dividida en clases a partir de una desigual distribución de recursos, lo que supone tensiones entre clases con intereses enfrentados, mecanismo fundante de la división socioespacial. Fals-Borda (1986) caracteriza a la participación como la ruptura voluntaria y vivencial de las relaciones asimétricas que conllevan sumisión y dependencia, y en este sentido es directamente extrapolable a las condiciones urbanas de fragmentación.

Los enfrentamientos y rupturas sociales se manifiestan en el territorio a partir de una sectorización espacial claramente perceptible, consolidada por la acción (o inacción) del Estado.

"...la estructura misma de la ciudad no ha permitido el desarrollo de mecanismos 
efectivos de participación de la Comunidad en la toma de decisiones (...), ya que el Estado concentra su acción y gestión de manera diferenciada dependiendo del tipo de interés que tenga para las distintas áreas de la ciudad, en correspondencia con los intereses de la administración central y la institución. En otras palabras no puede haber democratización ${ }^{8}$ política sin un cierto grado de democratización económica y social" (Duhau y Girola, 1990).

Se evidencia una relación dialéctica entre los procesos sociales de producción del espacio, cofundándose en una dinámica en la que uno limita y/o viabiliza al otro. Esta relación aporta los datos sobre las dinámicas locales, y permiten vincular causas y efectos en el proceso de conformación urbana. Esta conformación da cuenta de las condiciones concretas en las que se produce o no la participación.

La participación en la producción del hábitat es un proceso mediante el cual se emprende la mejora del hábitat -tanto tangible como intangible- de un grupo o comunidad a partir de la iniciativa vecinal. Se trata de un ejercicio activo y sostenido en el tiempo, mediante ámbitos y prácticas apropiadas y transformadoras, a través de las cuales se construyen vínculos interpersonales capaces de mantener el predominio de la integración comunitaria sobre la fragmentación (Boldrini, 2012).

Esta definición implica una serie de particularidades que dan cuenta de un ejercicio participativo pleno, incompleto o directamente frustrado.

En relación a los ámbitos en los que se desarrollan los vínculos y las modalidades de trabajo instrumentadas, se estructuran a partir del desarrollo de asambleas, gestiones en las

\footnotetext{
8 Por democratización se entiende "la progresiva nivelación de las posibilidades de acceso a los bienes societales, a la información necesaria para asumir actitudes racionalmente fundadas respecto de las decisiones colectivamente vinculantes y a la participación en la toma de decisiones" (Duhau, E., y Girola, L., 1990).
}

instituciones responsables, talleres, y también movilizaciones como mecanismo de protesta en caso de fracasar las gestiones diplomáticas entre la organización barrial y las entidades públicas responsables (Boldrini, 2012). Estos ámbitos pueden desarrollarse de diversas formas y orden, dando lugar a una gran variedad de casos.

Particularmente, la modalidad de protesta con movilizaciones se presenta como una de las acciones o instrumentos utilizados explicando el desencuentro comunidad-institución. Se utiliza para ejercer un mayor grado de presión con las demandas sociales, y a la vez se acerca a posibles transformaciones de carácter repolitizante, que buscan la ruptura de relaciones desiguales. Esta característica, propia de los procesos sociales emancipadores, es la que los diferencia de una protesta aislada, o una queja sin posibilidades de desarrollo y resolución.

Otro aspecto que debe cumplirse en el proceso para que este sea participativo es la construcción de vínculos endógenos a la organización, sin los cuales es imposible alcanzar la integración comunitaria a otra escala urbana (Boldrini, 2012).

La construcción de vínculos presenta una serie de variables internas que dan cuenta de su constitución, entre las que se encuentra la conformación de relaciones dialógicas. Si bien el modelo dominante se caracteriza por el desarrollo de relaciones dominadordominado, un proceso participativo implica el establecimiento de relaciones que intentan superar al modelo hegemónico. Este cambio conlleva la ruptura con roles instituidos y se cristaliza en el acceso concreto a la toma de decisiones, garantizada para todos los sujetos integrantes de la experiencia, tanto en la definición de la propuesta como en el surgimiento de nuevas demandas en relación con el objetivo principal. Las protestas explicitan la lucha por superar relaciones asimétricas a nivel exógeno, es decir, entre la comunidad y otro, frecuentemente el Estado a partir de la intervención de una institución en particular (Municipio, Instituto de Vivienda, entre otros).

El reclamo colectivo da cuenta tanto de la necesidad como de la capacidad de al- 
Figura $\mathrm{N}^{0} 1$

Esquema de los modos de acceso al hábitat digno

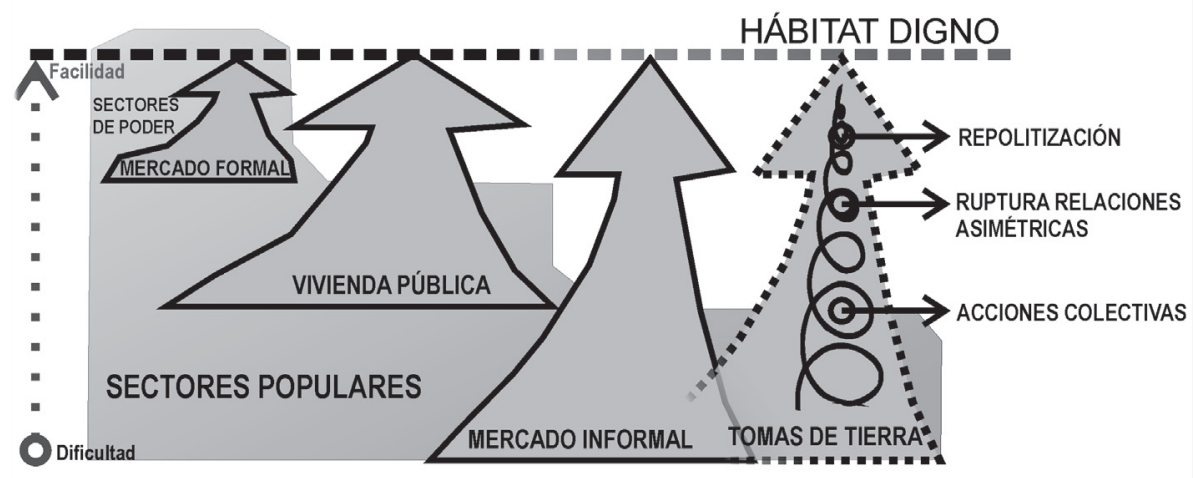

Fuente: Elaboración propia.

canzar la ruptura de relaciones desiguales. En este sentido, expresa la existencia de condiciones de subordinación entre los grupos enfrentados en el proceso. Es que si bien ambos aluden a la necesidad de confluencia de necesidades que contribuyan a superar estas desigualdades, actualmente predomina el desencuentro entre Comunidad y Estado. Mientras esta realidad se mantenga, la protesta será el instrumento visible de los procesos emancipadores (Boldrini, 2012).

La Figura $N^{\circ} 1$ muestra diversas situaciones en las que se produce el acceso al suelo y al hábitat digno. El extremo superior de la "pirámide social" corresponde a los sectores de poder en condiciones de facilidad plena para el acceso al hábitat mediante el mercado formal. En el extremo inferior o base de la pirámide social donde se encuentran los sectores populares, las condiciones de acceso son muy dificultosas debiendo recurrir al mercado informal o las tomas de tierras. En una situación intermedia se encuentra la clase media que es la principal beneficiada con la ayuda del Estado, puesto que hacia ella está dirigida la mayor parte del presupuesto destinado a políticas habitacionales.

\section{El Área Metropolitana de Tucumán}

San Miguel de Tucumán se fundó en el año 1685. Las sucesivas extensiones a la planta urbana que se realizaron fueron pro- longaciones hacia las cuatro orientaciones de las calles de la cuadrícula fundacional.

Desde inicio del siglo XX, la ciudad contó con una planta consolidada que hoy se conoce como el área central, junto a la conformación de villas o centros periféricos vinculados a la ciudad fundacional a través de las principales avenidas.

En esta área, hoy, la cobertura de servicios y equipamiento público es prácticamente completa, con una importante variedad socioeconómica de actividades.

A principios del siglo $\mathrm{XX}$ aparecieron las villas en las áreas circundantes a la ciudad, caracterizadas por una marcada identidad. Yerba Buena, por ejemplo, se desarrolla con vivienda de segunda residencia aglutinando sectores de elevado poder adquisitivo. La Banda del Río Salí al este, aglutina principalmente sectores de clase obrera próximos a los ingenios azucareros.

En la década de 1970 la ciudad fundacional conurbada con las villas circundantes, conforma el Gran San Miguel de Tucumán, y así es registrado por el censo nacional correspondiente. El proceso de metropolización que se inicia está marcado por un contexto de profunda crisis económica. A fines de los 60 las medidas macroeconómicas implementadas a nivel nacional repercutieron en Tucumán con el cierre de 20 ingenios azucareros, lo que produjo la migración de 200 mil 
personas de áreas rurales de la provincia a la ciudad de San Miguel y de allí al gran Buenos Aires. La consecuencia espacial fue un gran crecimiento urbano, mayoritariamente informal, dirigido hacia todas las direcciones en forma de anillos sucesivos que se han ido incorporando al casco fundacional vinculando a las villas circundantes.

La gran migración campo-ciudad a raíz de la crisis económica, es especialmente significativa en la evolución de la ciudad porque significó el surgimiento de un problema que hasta ese momento no tenía precedentes: la aparición de asentamientos informales. En la década de los 70 la ciudad reúne a 400 mil habitantes, de los cuales la séptima parte (58 mil personas) presentan problemas de hábitat. El trabajo realizado por el arquitecto Roberto Gómez López (1976) realiza un relevamiento de asentamientos en la ciudad de 1970. En dicho relevamiento se distinguen 108 asentamientos, entre "marginales" sin intervención del Estado y "de mejora" en los que, mediante fondos públicos, se han introducido mejoras que suponen la superación de la transitoriedad y vulnerabilidad extrema propias del primer tipo.

La conformación del AMET como área metropolitana se desarrolló tal como lo expresa la Figura $\mathrm{N}^{0} 2$, a partir de la anexión de nuevo suelo urbano. El principal crecimiento se produjo a ambos lados del eje de circulación principal este-oeste que atraviesa la ciudad de lado a lado conectando el río con la cadena montañosa del Aconquija.

El proceso de metropolización estuvo marcado por la conurbación entre las vecinas ciudades de Banda del Río Salí, Alderetes, Yerba Buena y Tafí Viejo y Yerba Buena sumado al incipiente desarrollo de nuevas centralidades configuradas a partir de la construcción de vivienda de interés social en Las Talitas, El Manantial y Cebil Redondo. El nuevo suelo urbano no fue convenientemente dotado de la infraestructura y el equipamiento urbano básico (Gómez López, 2000).

Con la llegada del neoliberalismo se implementan políticas y comportamientos sociales que producen nuevas lógicas en el desarrollo residencial, ahora claramente desarticulado como consecuencia de la profunda fragmentación social imperante. La periferia crece expansivamente pero de manera desigual, con municipios que se desarrollan y constituyen exclusivamente a partir de la promoción de vivienda pública, otros con asentamientos informales y vivienda de promoción estatal, mientras que otros concentran la urbanización de lujo con barrios cerrados, countries y viviendas de alto nivel (Gómez López et al., 2012).

Los bordes suburbanos, cada vez menos definidos e inconclusos, se conforman con un repertorio tipológico que va desde asentamientos informales, viviendas de promoción pública, urbanizaciones cerradas, además de
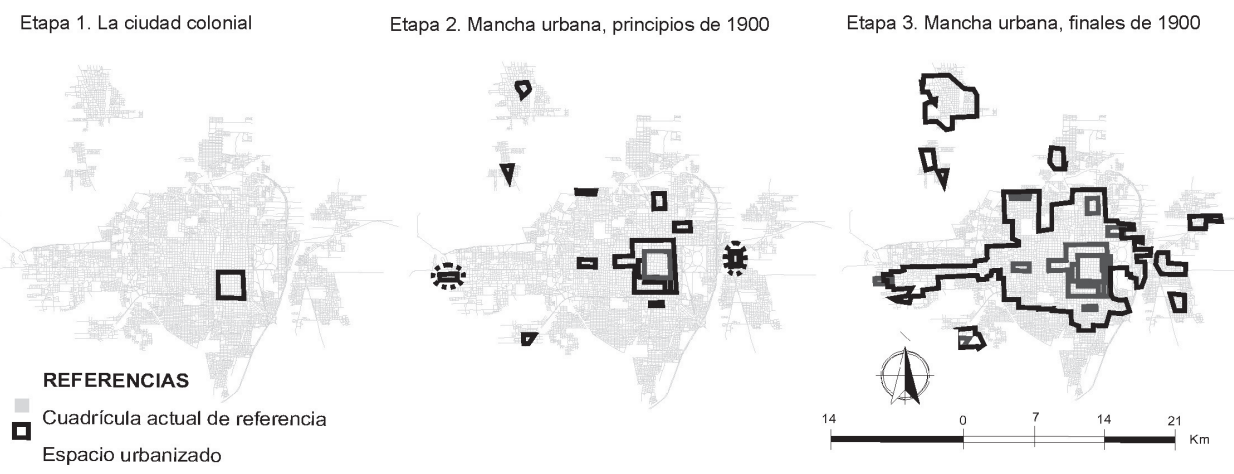

Fuente: Boldrini (2012) en base a mapas del IPDU-FAU-UNT. 
las tipologías tradicionales de vivienda en planta baja y altura. El uso residencial en el área de crecimiento presenta en general una muy escasa diversidad morfológica de usos (Gómez López, 2000).

Es importante destacar que el crecimiento de la ciudad se produce en un marco de carencia de planeamiento urbano donde se definan criterios de diseño y usos de suelo de las áreas de expansión, en un contexto de ejecución de políticas aisladas que facilitan el desarrollo de proyectos privados, apoyados en la falta de planificación y control público.

\section{Expansión residencial en el Área Metropolitana de Tucumán}

A partir de la localización de diferentes grupos sociales en el aglomerado de acuerdo a tipologías residenciales reconocidas en la ciudad, se analiza la distribución socioeconómica de los mismos en el aglomerado, haciendo hincapié en el área de expansión 1976-20089 y particularmente en la dinámica desarrollada por los sectores populares ${ }^{10}$.

Los tipos residenciales fueron localizados de acuerdo a la siguiente categorización (Gómez López et al., 2012) ${ }^{11}$ en la que se privilegia la identificación espacial de los grupos pertenecientes a las franjas extremas de la sociedad, tales como las urbanizaciones

9 La selección de los años se debe a que la década de 1970 representa la etapa de mayor crecimiento demográfico del Área Metropolitana derivada del masivo aumento las migraciones campo - ciudad como resultado del cierre de 11 ingenios en la Provincia (Pucci, 2007). La intención es comparar esa ciudad recientemente constituida como área metropolitana, con las condiciones actuales del aglomerado.

10 Para esto se trabajó con las imágenes satelitales del AMET en soporte GIS 3.3, y haciendo un avance sobre la investigación realizada por Gómez López, Boldrini y Cuozzo en 2012.

11 Estos mismos tipos residenciales (1, 2 y 3 ) fueron reconocidos tanto por sus características materiales como por su origen y relación con el medio urbano en el Informe Geo 2007 (Geo, 2007: 89-90), pero aún con escaso impacto del tercer tipo, situación que hoy ha cambiado como se verá más adelante. cerradas $^{12}$, las urbanizaciones informales ${ }^{13} y$ los barrios de promoción pública ${ }^{14}$. Asimismo, existe suelo urbano no tipificado, que compromete tipologías tradicionales con un uso más heterogéneo por parte de los diferentes sectores sociales, así como áreas verdes, espacio público y equipamiento comunitario de toda clase.

La Figura $N^{\circ} 3$ presenta la organización administrativa que comprende el Área Metropolitana de Tucumán, y la Figura $N^{\circ} 4$ muestra la localización de las tipologías antes desarrolladas en la mancha urbana del aglomerado correspondiente al año 2008.

Figura $\mathrm{N}^{\mathrm{o}} 3$

Organización administrativa del AMET

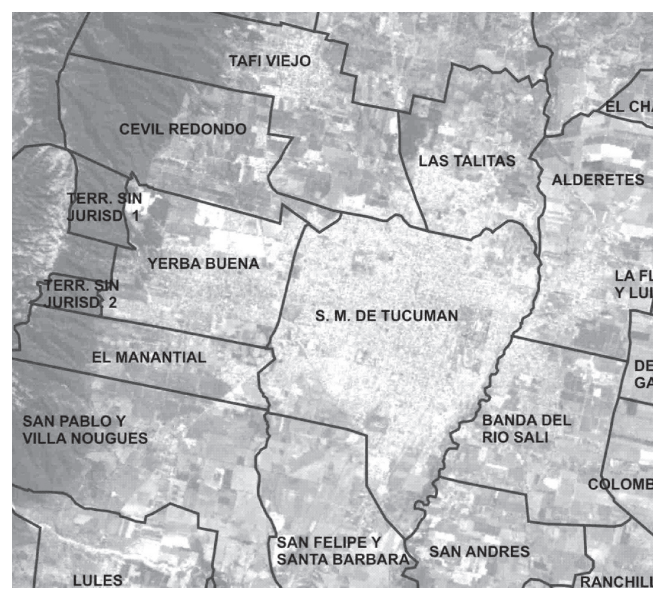

Fuente: Gómez López et al., 2012.

12 Se refiere a countries y barrios cerrados como residencia de sectores de clase alta y media-alta. Se trata de porciones de la ciudad, cerradas en polígonos de baja densidad. Son emprendimientos privados de alta calidad ambiental y paisajística.

${ }^{13}$ Son aquellos barrios de crecimiento informal y condiciones de urbanización incompleta fundamentalmente por carencia de infraestructuras básicas. El proceso de urbanización empieza por la ocupación de los terrenos, sigue con la delimitación autogestionada de lotes, la gestión vecinal de infraestructuras y servicios, acompañado por la lenta y progresiva construcción de las viviendas precarias, por último la gestión para la regularización dominial y en el mejor de los casos y en forma gradual el mejoramiento barrial.

${ }^{14}$ En estos casos la urbanización es planificada, corresponde a los barrios ejecutados por el Instituto Provincial de la vivienda u otra entidad estatal como el Banco Hipotecario, la Caja Popular de Ahorros, etc. Se trata de áreas que se urbanizan en forma de polígonos, y en ellas el loteo, urbanización y construcción se hacen conjuntamente. 


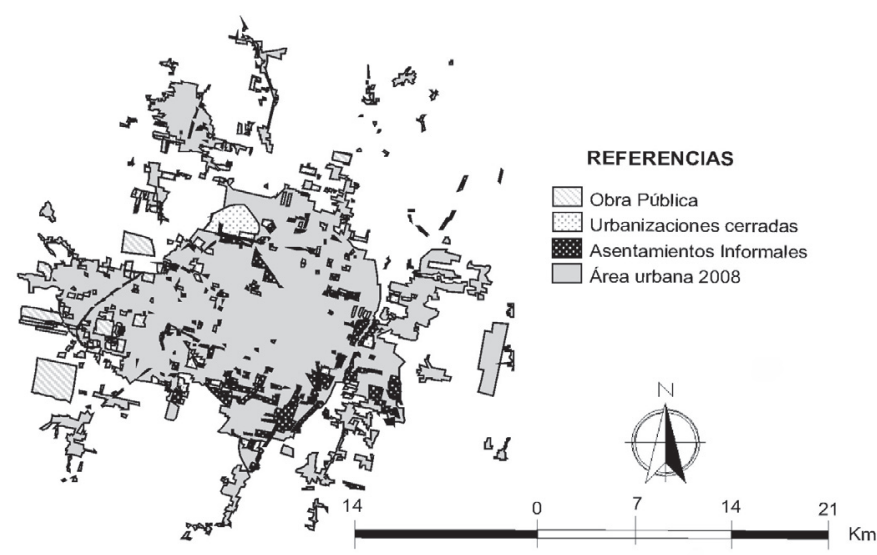

Fuente: Gómez López et al., 2012.

En la Figura $\mathrm{N}^{\circ} 4$ es posible visualizar el Área Metropolitana de Tucumán en el año 2008. En el sentido este y extendiéndose hacia el sur de la ciudad capital, el crecimiento urbano se desarrolló dando prioridad y hasta casi exclusividad a las tipologías populares, como el municipio Banda del Río Salí, caracterizado por la presencia del río Salí altamente contaminado, así como el sur de la capital considerada un área con riesgo ambiental por inundación. Hacia el oeste, en los municipios de Yerba Buena y la comuna de El Manantial, el uso residencial se desarrolló fundamentalmente mediante la proliferación de urbanizaciones privadas, ligado al valor ambiental que dicho espacio posee por su estrecha vinculación con la sierra de San Javier. Las principales administraciones donde se alojan las obras públicas son en la comuna de Cevil Redondo y el municipio de Tafí Viejo (Gómez López et al., 2012). Esta última localidad aloja el megaemprendimiento habitacional Lomas de Tafí, con 5.500 viviendas producidas mediante la obra pública.

\section{Expansión urbana y problemas ambientales en el aglomerado}

A fin de avanzar en las variables que hacen al tema de investigación, el mapa de ex- pansión urbana se presenta en correspondencia con un mapa de problemas ambientales del territorio que ocupa el AMET (Malizia y Boldrini, 2012 $)^{15}$, dando cuenta de la estrecha relación entre precariedad habitacional y vulnerabilidad ambiental, evidenciadas en las Figuras $\mathrm{N}^{\circ} 5$ y $\mathrm{N}^{\circ} 6$.

Cruzando esta información en un entorno de trabajo SIG, se cuantificó la influencia de los problemas ambientales para cada tipología residencial. La cantidad de suelo urbano afectado por estos problemas para el total del AMET asciende al 35,04\% de su superficie, tal como indica el Cuadro $N^{\circ} 1$. Asimismo, esta problemática ambiental afecta al $90,37 \%$ de los barrios informales, al 54,54\% de barrios cerrados y al $49,12 \%$ de barrios producidos por el Estado a través del Instituto Provincial de Vivienda.

Los barrios informales del AMET ocupan los sectores de la ciudad que los grupos inmobiliarios descartan. Estos son los espacios de baja calidad ambiental, escasa o nula

\footnotetext{
15 Para la elaboración del plano de problemas ambientales del AMET se usó como referencia el trabajo de Sayago, Collantes, Neder y José Busnelli (2010).
} 
Figura $\mathrm{N}^{\circ} 5$

Expansión urbana 1976-2008 con clasificación residencial

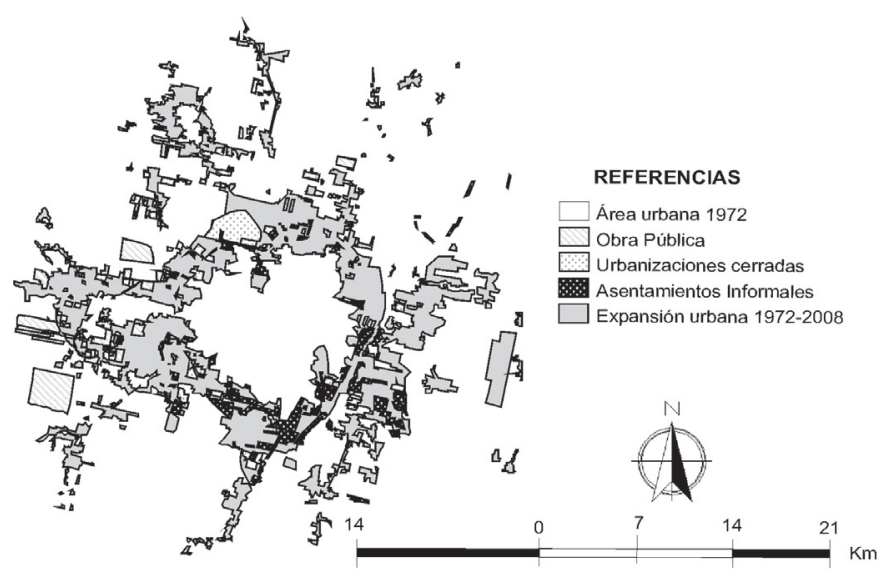

Fuente: Gómez López et al., 2012.

Figura $\mathrm{N}^{\circ} 6$

Problemas ambientales del territorio que ocupa EL AMET

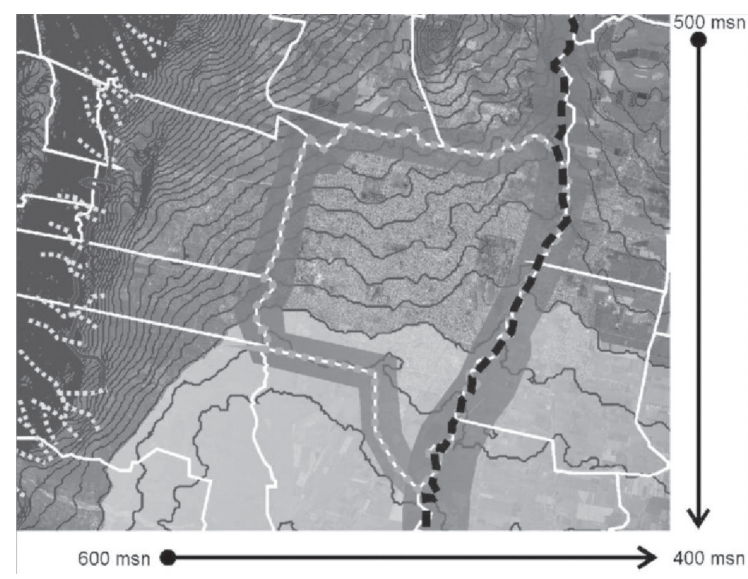

Referencias

I - Río Salí área de inundación

I E Canales urbanos área de inundación

- Abanicos fluviales baja capacidad de soporte del suelo

- Cotas de nivel cada $10 \mathrm{~m}$

- Área de amortiguamiento

- División Jurídica

Áreas inundables por desborde de rios y canales Áreas inundables por cota de nivel baja

Fuente: Malizia y Boldrini, 2012

capacidad de soporte urbano, que requieren costosas obras para su saneamiento. La mayor parte de las urbanizaciones informales se encuentran en áreas de riesgo, principalmente inundables y contaminadas.

Si bien todas las tipologías residenciales tienen casos mal localizados, los porcentajes son mucho menores en el caso de las urba- nizaciones cerradas y la obra pública. Las urbanizaciones cerradas se localizan sobre la zona pedemontana de elevada calidad ambiental. Sin embargo, también estas áreas tiene riesgo de inundación, desmoronamiento o tienen la presencia de canales urbanos secundarios considerados perjudiciales, aunque en estos casos específicos se encuentran mejor canalizados, disminuyendo los riesgos 
Cuadro $\mathrm{N}^{\circ} 1$

Superficie residencial afectada por problemas ambientales

\begin{tabular}{|l|r|r|r|r|}
\hline \multicolumn{1}{|c|}{ Categoría } & $\begin{array}{c}\text { Sup. total } \\
\text { (ha) }\end{array}$ & $\begin{array}{c}\text { Sup. afectada } \\
\text { (ha) }\end{array}$ & $\begin{array}{c}\text { Porcentaje de cada } \\
\text { tipología en el } \\
\text { AMET }\end{array}$ & $\begin{array}{r}\text { Porcentaje de afección } \\
\text { ambiental para cada } \\
\text { tipología residencial }\end{array}$ \\
\hline $\begin{array}{l}\text { Asentamientos } \\
\text { informales }\end{array}$ & $1.154,8$ & $1.043,57$ & 8,4 & 90,37 \\
\hline $\begin{array}{l}\text { Urbanizaciones } \\
\text { cerradas }\end{array}$ & $1.060,23$ & 578,23 & 3,7 & 54,54 \\
\hline Obra pública & 929,95 & 456,81 & 35,0 & 49,12 \\
\hline Sup. urbana total & $12.455,46$ & $4.364,60$ & 35,04 \\
\hline
\end{tabular}

Fuente: Elaboración propia sobre la base de mapas de Gómez López et al., 2012. Base de datos proyecto CIUNT 2008-2010

de desborde. También ocupan sectores con problemas de soporte del suelo como áreas pedemontanas. Estos problemas pueden contrarrestarse con la construcción de obras civiles que estos grupos pueden solventar. Es significativo el impacto ambiental que estos emprendimientos producen al no contar con cloacas o infraestructuras para el saneamiento de los líquidos cloacales que se eliminan a pozo ciego, contaminando un área de recarga de acuíferos.

También hay que señalar que un gran número de barrios construidos por el Instituto Provincial de Vivienda y Desarrollo Urbano (IPV y DU) se localiza en suelo sin aptitud para urbanización ya sea por riesgo de inundación, de contaminación por contacto con cauces contaminados como el del río Salí o por el desborde hídrico de canales próximos. Estos mismos inconvenientes afectan a una importante superficie ocupada por barrios del IPV y DU, que se localizan en suelo no apto para urbanización por presentar alguno de estos tipos de riesgo ambiental.

\section{Participación de los sectores populares en la dinámica urbana del aglomerado}

La participación de los sectores populares en la dinámica urbana se reconoce a partir de la comparación entre las condiciones de la localización de los barrios informales y obras públicas para sectores de escasos recursos correspondientes al año 1976 (Gómez López, 1976) ${ }^{16}$, con las condiciones de localización del mismo tipo residencial en el año $2008^{17}$.

La urbanización popular se manifiesta a partir del reconocimiento de viviendas precarias, carencia de servicios y/o ocupación de sectores sin habitabilidad básica ${ }^{18}$. Estos barrios se encuentran desarticulados de la trama urbana consolidada, a partir de la cercanía o incluso la superposición con bordes urbanos

\footnotetext{
16 Los datos disponibles asumen a los sectores populares como grupos marginales, lo que incluye según el autor (Gómez López, 1976) tanto asentamientos irregulares como obras del IPV y DU. Es por esto que la comparación se realiza teniendo en cuenta los sectores populares como aquellos grupos sociales pertenecientes a la clase social de bajos o muy bajos ingresos, que se manifiestan en el territorio localizados en barrios sin consolidar y predominantemente desarticulados de la trama urbana consolidada.

17 No se considera obra pública del Estado para el año 2008, ya que requeriría la diferenciación entre programas destinados a sectores de recursos escasos y medios.

18 "...estos sectores (socioeconómicamente bajos y muy bajos) se caracterizan por la precariedad de las construcciones y por la falta de una calidad de vida adecuada. La falta de servicios próximos a su vivienda, el costo del transporte público, sumado a la desconexión de las áreas centrales, implican un doble perjuicio para los sectores populares y a la vez una mayor dependencia del centro" (Di Lullo y Giobellina, 1996:95).
} 
que contribuyen a separarlos de otros sectores, limitando físicamente la comunicación (Ariñez et al., 2003). Desde el punto de vista subjetivo, son negados por la ciudad, atribuyéndoles un carácter peligroso, criminalizándolos (Sabatini y Brain, 2008).

De la comparación de los cortes temporales 1976 y 2008, expresados en el Cuadro $N^{\circ}$ 2 y la Figura $N^{\circ} 7$, se detectan:
- Asentamientos informales que existen en el año 1976 y aparecen consolidados en el año 2008.

- Asentamientos informales que existen en el año 1976 y se mantienen como tales en el año 2008.

- Nuevos de asentamientos informales.

Resulta significativo en el proceso de expansión metropolitana el incremento del área

Cuadro $\mathrm{N}^{\circ} 2$

Superficies ocupadas por asentamientos informales 1976-2008

\begin{tabular}{|c|c|c|c|c|}
\hline Localidad & $\begin{array}{c}\text { Asentamientos } \\
\text { informales } 1976 \\
\text { (ha) }\end{array}$ & $\begin{array}{l}\text { Asentamientos } \\
\text { informales que se } \\
\text { mantienen hasta } \\
2008\end{array}$ & $\begin{array}{l}\text { Asentamientos } \\
\text { informales } \\
\text { consolidados en } \\
2008 \text { (ha) }\end{array}$ & $\begin{array}{l}\text { Asentamientos } \\
\text { informales nuevos } \\
\text { en } 2008 \text { (ha) }\end{array}$ \\
\hline Alderetes & 14,27 & 0 & 14,27 & 14,27 \\
\hline Banda del Río Salí & 12,12 & 0 & 12,12 & 93,22 \\
\hline Cevil Redondo & 0 & 0 & 0 & 6,30 \\
\hline El Manantial & 0 & 0 & 0 & 12,86 \\
\hline Las Talitas & 8,12 & 0 & 8,12 & 25,71 \\
\hline Los Nogales & 0 & 0 & 0 & 0 \\
\hline San Felipe & 0 & 0 & 0 & 2,78 \\
\hline $\begin{array}{l}\text { San Miguel de } \\
\text { Tucumán }\end{array}$ & 231,03 & 67,83 & 154,61 & $1.148,25$ \\
\hline $\begin{array}{l}\text { San Pablo, Villa } \\
\text { Nougues }\end{array}$ & 0 & 0 & 0 & 0 \\
\hline Tafí Viejo & 22,70 & 12,34 & 10,36 & 46,83 \\
\hline Yerba Buena & 41,48 & 3,61 & 37,86 & 55,83 \\
\hline Totales & 329,74 & 83,80 & 237,36 & $1.406,03$ \\
\hline
\end{tabular}

Fuente: Boldrini, 2012.

Figura $\mathrm{N}^{0} 7$

Gráfico comparativo de asentamientos informales 1976-2008

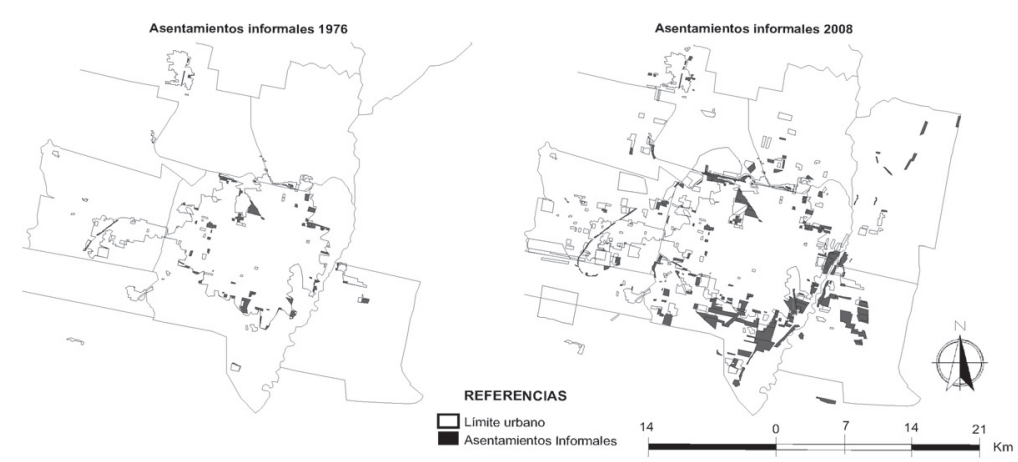

Fuente: Boldrini, 2012. 
ocupada por sectores populares que pasó del $6 \%$ del suelo metropolitano en 1976 a casi el $20 \%$ en el 2008.

Cabe destacar que el $71,9 \%$ de los espacios ocupados por sectores populares del año 1976, aparecen como consolidados en el año 2008. En el 2008 se observan barrios informales en tres localidades que no tenían urbanización sin consolidar en el año 1976.

A fin de profundizar el conocimiento sobre los barrios "antes informales" que hoy se muestran consolidados, se realizó un muestreo del $50 \%$ de los doce barrios identificados en forma de polígonos aislados, corroborando el estado actual de infraestructura, viviendas y antigüedad de los vecinos, a fin de detectar posibles migraciones urbanas internas.

El análisis mostró que la movilidad es poco significativa. Los vecinos de la década de 1970 son los que habitan barrios ahora consolidados; sin embargo, se trata de barrios que si bien han progresado en términos físicos (con más y mejores servicios, algunas caIles pavimentadas y viviendas consolidadas), permanecen desarticulados de la ciudad. Se trata de sectores que habiendo mejorado considerablemente su calidad urbana, siguen perteneciendo a familias de escaso poder adquisitivo, discriminadas socialmente $y$, por ende, marginados de la trama sociourbana consolidada.

\section{Las antípodas residenciales}

Las nuevas urbanizaciones informales se ubican principalmente en la capital, alcanzando las 1.148,25 ha, lo que representa un $81,6 \%$ del total de asentamientos existentes en el AMET, y un aumento de esta tipología de casi un $500 \%$ dentro de esta localidad. Cabe acotar que la capital abarca el $44 \%$ de la superficie del aglomerado metropolitano.

En segundo orden le sigue la localidad de la Banda del Río Salí con 93,2 ha de barrios informales, que supone el 6,6\% del total del AMET, y un crecimiento interno de un $760 \%$ de esta tipología, localizada básicamente sobre las márgenes del río contaminado.
En tercer orden, en cantidad de barrios informales, sigue Yerba Buena con 55,8 ha, que representa el 3,9\% de la superficie de urbanización popular del AMET. Sin embargo, Yerba Buena es la única localidad en la que los barrios informales no crecen espacialmente entre los años 1976 y 2008, solo se observan relocalizaciones, siendo el ámbito preferido para las urbanizaciones cerradas.

Tafí Viejo sigue en el orden decreciente, con 46,83 ha ocupadas por barrios informales, que representan un 3,3\% del total de asentamientos de estas características en el AMET. Sin embargo, lo que caracteriza esta localidad es el explosivo crecimiento de los barrios de promoción pública por la ejecución del megaemprendimiento Lomas de Tafí, con 5.500 nuevas viviendas.

De esta forma se observa como el crecimiento urbano popular cambia desde el año 1976, pasando de un "cinturón de pobreza" alrededor de la capital, a un aglomerado sectorizado en municipios ricos y pobres, en perfecta consonancia con la oferta del mercado inmobiliario acorde a la calidad ambiental que los mismos ofrecen.

\section{Las tomas de tierra en el aglomerado tucumano}

Se reconoce a las tomas de tierra como una de las expresiones más impactantes de la carencia de hábitat, cuyas consecuencias interpelan a la sociedad en general y requieren de la intervención del Estado. Sin embargo, las mismas aparecen en periódicos locales al momento de la toma de predios, pero desaparecen del ámbito mediático al poco tiempo.

La Figura $N^{\circ} 8$ muestra tomas de tierras registradas a partir del relevamiento de las fuentes periodísticas locales. Las mismas se localizan en áreas próximas a obras públicas, buscando llamar la atención del Estado. Las tomas de tierra que se producen al sur de la ciudad, no se identifican como conflictivas en tanto se trata de áreas de escaso valor ambiental, despreciadas por el mercado inmobiliario. 
Figura $\mathrm{N}^{\circ} 8$

Tomas de tierra en el AMET

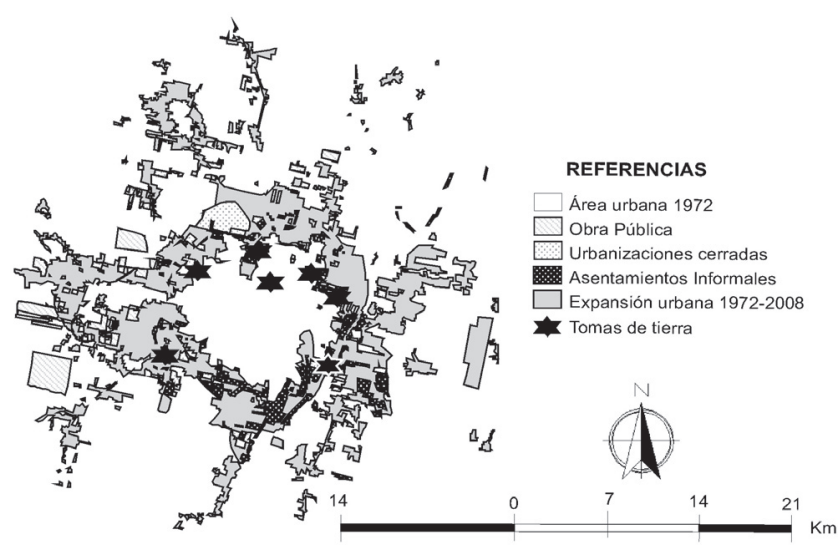

Fuente: Elaboración propia.

\section{Conclusiones}

El Área Metropolitana de Tucumán crece en un $140 \%$ entre 1976 y el año 2008. La incidencia del suelo ocupado por urbanizaciones informales crece más significativamente pasando de un 6\% del suelo metropolitano a casi el $20 \%$ del mismo. La brecha social es cada vez mayor. Si bien en el periodo considerado el crecimiento espacial del hábitat informal es casi tan importante como el de las urbanizaciones cerradas, la población involucrada en el primero es cuatro veces mayor. Los barrios informales más recientes se localizan en áreas sin aptitud para urbanización con riesgos ambientales, descartadas por el mercado inmobiliario formal.

Comparando temporalmente el proceso de urbanización popular se observa que los barrios informales de 1976, a pesar de presentarse como sectores consolidados en el 2008, mantienen condiciones de vulnerabilidad y desarticulación respecto a la trama urbana actual. Esto pone en evidencia que la problemática habitacional supera los registros oficiales, junto al testimonio de la falta de recursos (materiales e inmateriales) que poseen estos sectores para superar la inercia de la condición de privación original.
La ciudad refleja la desigualdad. Su estudio y análisis resulta invaluable para encuadrar a los sectores populares en el mapa de relaciones socioespaciales donde desarrollan sus dinámicas barriales cotidianas, punto de partida para profundizar los estudios sobre los modos de participación existentes. Esto permite comprender a procesos de urbanización popular como invasiones o tomas de tierras, como implosiones sociales y espaciales producto de la organización desigual del espacio y no como fenómenos externalizantes.

Las tomas de tierra, como fenómeno inherente a la protesta social, son emergentes de insurrección popular, vinculados específicamente a la producción del hábitat. Mediante acciones concretas en relación al acceso al suelo urbano y aún más cuando se ven acompañadas de un crecimiento socioorganizativo y otros aspectos inherentes a la participación (Boldrini, 2012), representa un acto de emancipación en el actual contexto de desigualdad sociourbana.

Por último, si bien las tomas de tierra parecen aún no haber despertado en el aglomerado tucumano -al menos en relación a la magnitud actual del problema habitacional-, los antecedentes sugieren un aumento de este 
proceso de urbanización informal. Esto será así en tanto el Estado no asuma una política de provisión de suelo urbano en la que se garantice la articulación del suelo producido con la ciudad consolidada formal.

\section{Referencias bibliográficas}

ARIÑEZ, N.; BOLDRINI, P. y LASKOWSKI, C. Propuesta de consolidación urbana para San Miguel de Tucumán, desde la ciudad de la pobreza. Tucumán: Tesina para Facultad de Arquitectura y Urbanismo de la Universidad Nacional de Tucumán, 2003.

BOLDRINI, P. Producción participativa del hábitat popular en el área metropolitana de Tucumán. Tucumán: Tesis para optar al grado de Doctor de la Facultad de Filosofía y Letras de la Universidad Nacional de Tucumán, 2012.

BOMBA, H.; CASARES, M.; DI LULLO, R. y CAMINOS, R. Escenarios GEO San Miguel de Tucumán. Perspectivas del medio ambiente urbano. Pampa, 2007, N³ 3, p. 75-89.

BORJA, J. Ciutadania i espai públic. En: SUBIRÓS, P. (editor). Ciutat real, ciutat ideal, Debat de Barcelona (III). Barcelona: Centre de Cultura Contemporània de Barcelona, 1998, p.43-59.

CLICHEVSKY, N. Construcción y administración de la ciudad latinoamericana. Buenos Aires: Instituto Internacional de Medio Ambiente y Desarrollo, 1990.

DAVIS, M. Planeta de Ciudades Miseria. Madrid: Foca Ediciones, 2007.

DI LULLO, R. y GIOBELLINA, B. La otra ciudad: Tucumán frente al 2000. Tucumán: Universidad Nacional de Tucumán, 1996.

DUHAU, E. y GIROLA, L. La ciudad y la modernidad inconclusa. Revista Sociológica, 1990, Vol. 5, No 12, p. 9-31.

FALS-BORDA, O. La investigación-acción participativa: Política y epistemología. En: CAMACHO, A. (editor). La Colombia de hoy. Bogotá: Cerec, 1986, p. 21-38.
FERNÁNDEZ, R. Interrogantes sobre la sustentabilidad de la política habitacional Argentina. Córdoba: Seminario Iberoamericano de Ciencia y Tecnología para el Hábitat Popular, 2006.

GÓMEZ LÓPEZ, C. Asentamientos marginales de vivienda en San Miguel de Tucumán. Tucumán: Universidad Nacional de Tucumán, 1976.

GÓMEZ LÓPEZ, C. Influencia de la Promoción pública de vivienda en el desarrollo de la mancha urbana de la ciudad de San Miguel de Tucumán. Elementos para la comprensión de su estructura urbana. Valencia: Universidad Politécnica de Valencia, 2000.

GÓMEZ LÓPEZ, C.; CUOZZO, R. y BOLDRINI, P. Los usos del suelo en el entorno metropolitano. Cambios y tendencias. En: GRAU, R. San Javier y el área metropolitana de San Miguel de Tucumán: Dinámica de una interfase natural-urbana. Tucumán: Universidad Nacional de Tucumán, 2006, p. 167-190.

GÓMEZ LÓPEZ, C.; CUOZZO, R. y BOLDRINI, P. Nuevas lógicas de expansión en la dinámica urbana de una ciudad intermedia. El caso del aglomerado tucumano en el norte argentino. Tucumán: Grupo de Universidades Montevideo, 2012.

INDEC. Censo Nacional de Población y Vivienda 2001 Resultados provisionales total del país. Buenos Aires: INDEC, 2001.

JANOSCHKA, M. El nuevo modelo de la ciudad latinoamericana: fragmentación y privatización. EURE, 2002, Vol. 28, № 85, p. 11-20.

LÓPEZ, E. Políticas habitacionales e intervención social. Revista Margen, 2002, № 26. Disponible en internet: http://www.margen. org/suscri/margen26/ficha.html

MALIZIA, M. y BOLDRINI, P. Las lógicas de ocupación del espacio urbano. Un estudio de realidades contrapuestas. El caso de las urbanizaciones cerradas y villas miseria en Yerba Buena, Gran San Miguel de Tucumán. Revista Cuadernos, 2012 (en prensa). 
PRÉVÔT SCHAPIRA, M. Segregación, fragmentación, secesión. Hacia una nueva geografía social en la aglomeración de Buenos Aires. Economía, Sociedad y Territorio, 2000, No 7, p. 405-431.

PRÉVÔT SCHAPIRA, M. Fragmentación espacial y social: conceptos y realidades. Perfiles Latinoamericanos, 2001, No 19, p. 33-56.

PUCCI, R. Historia de la destrucción de una Provincia. Buenos Aires: Ediciones Del Pago Chico, 2007.

QUINTAR, A.; CRAVINO, M. y GONZÁLEZ, M. Participación y política. Discutiendo algunos sentidos y prácticas participativas en América Latina. Otra Economía, 2010, Vol. 5, № 9, p. 116-125.

SABATINI, F. La segregación social del espacio en las ciudades de América Latina. Serie Azul № 35, 2003, p. 59-70.

SABATINI, F. y BRAIN, I. La segregación, los guetos y la integración social urbana: mitos y claves. EURE, 2008, Vol. 34, № 103, p. 5-26.
SAYAGO, J.; COLLANTES, M.; NEDER, L. y BUSNELLI, J. Cambio climático y amenazas ambientales en el Área Metropolitana de Tucumán, Revista de la Asociación Geológica Argentina, 2010, Vol. 66, N 4, p. 544-554.

TORRE, J.C. El proceso político de las reformas económicas en América Latina. Buenos Aires: Paidós, 1998.

VINUESA, J. Sobre el concepto de área metropolitana. Estudios geográficos, 1975, № 68, p. 140-41.

YORY GARCÍA, C. Topofilia, ciudad y territorio: una estrategia pedagógica de desarrollo urbano participativo con dimensión sustentable para las grandes metrópolis de América Latina en el contexto de la globalización: el caso de la ciudad de Bogotá. Madrid: Tesis doctoral de la Universidad Complutense de Madrid, 2003. 